\title{
Downregulation of CLCA4 expression is associated with the development and progression of colorectal cancer
}

\author{
LIHUI WEI $^{1,2^{*}}$, WUJIN CHEN $^{3 *}$, JINYAN ZHAO $^{1,2}$, YI FANG $^{1,2}$ and JIUMAO LIN ${ }^{1,2}$ \\ ${ }^{1}$ Academy of Integrative Medicine and ${ }^{2}$ Fujian Key Laboratory of Integrative Medicine on Geriatrics, \\ Fujian University of Traditional Chinese Medicine, Fuzhou, Fujian 350122; ${ }^{3}$ Department of Oncology, \\ Affiliated People's Hospital of Fujian University of Traditional Chinese Medicine, Fuzhou, Fujian 350004, P.R. China
}

Received August 26, 2019; Accepted April 1, 2020

DOI: $10.3892 / \mathrm{ol} .2020 .11640$

\begin{abstract}
The molecular mechanisms involved in the development and progression of colorectal cancer (CRC) are not completely understood. The present study aimed to identify potential novel genes involved in the development and progression of CRC. Database analysis revealed that the mRNA level of the chloride channel accessory 4 (CLCA4) was frequently lower in primary tumor tissues compared with that in corresponding non-cancerous colon tissues, and was even lower in liver metastases than in primary tumors. Further analyses through The Human Protein Atlas (THPA) website and immunohistochemistry (IHC)-based tissue microarray (TMA) confirmed that CLCA4 mRNA and protein expression were downregulated in CRC tissues. Furthermore, IHC-based TMA analysis revealed a gradual decrease in CLCA4 protein expression among colorectal normal, adenoma and carcinoma tissues. Survival analysis revealed that the decrease in CLCA4 mRNA expression was associated with the overall survival rate of patients with different types of tumor, including CRC, breast cancer, head and neck cancer and stomach cancer. Overall, downregulated CLCA4 expression may influence the development and progression of CRC.
\end{abstract}

Correspondence to: Dr Jiumao Lin, Academy of Integrative Medicine, Fujian University of Traditional Chinese Medicine, 1 Qiuyang Road, Minhou Shangjie, Fuzhou, Fujian 350122, P.R. China

E-mail: linjiumao@fjtcm.edu.cn

${ }^{*}$ Contributed equally

Abbreviations: CRC, colorectal cancer; CLCA4, chloride channel accessory 4; GEPIA, Gene Expression Profiling Interactive Analysis; THPA, The Human Protein Atlas; TMA, tissue microarray

Key words: colorectal cancer, CLCA4, prognosis, survival, development

\section{Introduction}

Colorectal cancer (CRC) is the third most common cancer worldwide (1-4). In 2019, there were almost 145,600 newly diagnosed patients, and more than 50,000 patients died from the disease in the United States (4). Surgery and adjuvant chemotherapy are the main treatments for CRC. The 5-year survival rate following surgical resection of colorectal metastases has increased from 25 to $55 \%$, but most patients relapse $(5,6)$. Therefore, further investigation on the underlying mechanisms involved in the development and progression of CRC may provide novel directions for CRC treatment.

Calcium-activated chloride channel (CLCA) regulators are proteins with a symmetrical homocysteine motif in the terminal tail of the amino group (7). The loci of the human CLCA genes are at chromosome 1p31-1p22 (8). Research has demonstrated that CLCA protein members influence a wide range of biological processes, including cell differentiation, adhesion, apoptosis and airway inflammation (7-9). Recent studies have revealed that the expression levels of CLCA proteins, such as CLCA1, CLCA2 and CLCA4, are abnormal in a variety of cancer types (10-16), and could therefore be potential cancer predictors for patients. CLCA1 inhibits the proliferation of CRC cells and has been associated with a favorable prognosis of patients with CRC (10). CLCA2 is a p53-inducible inhibitor of cell proliferation and could be a marker of differentiated epithelium that is downregulated with tumor progression (11). Low CLCA2 expression promotes cell proliferation and metastasis by driving the epithelial-to-mesenchymal transition signaling pathway $(12,13)$. CLCA4 has a similar structure to CLCA1 and CLCA2 (17), and loss of CLCA4 expression has been observed in hepatocellular carcinoma (14), breast cancer (15) and bladder cancer (16), facilitating tumor cell growth and metastasis via epithelial-to-mesenchymal transition. However, to the best of our knowledge, the role of CLCA4 in the prognosis of patients with CRC has not been well clarified. The present study aimed to measure the expression levels of CLCA4 during the development of $\mathrm{CRC}$ and assess its association with the survival of patients with different types of tumor, including CRC.

\section{Materials and methods}

Microarray data information and CLCA4 gene identification. The gene chip detection technique has been employed for more 
than 10 years (18). The National Center for Biotechnology Information (NCBI)-Gene Expression Omnibus (GEO) is a free database of microarray/gene profiles (https://www.ncbi. nlm.nih.gov/geo). For the present study, an original microarray dataset, GSE49355 (19), was downloaded from the NCBI-GEO database. The microarray data of GSE49355 is based on the GeneChip Human Genome U133 and includes 21 patients with advanced CRC (submission date: September 1, 2013).

Oncomine platform analysis. The transcriptional levels of CLCA4 in CRC specimens and normal controls were analyzed using the Oncomine platform (https://www.oncomine.org) (20). Additionally, the threshold of the P-values, fold-change and gene bank in cancerous tissues compared with non-cancerous type-matched tissues were evaluated.

GEPIA analysis. As an interactive web that includes 9,736 tumors and 8,587 normal samples from TCGA and the GTEx projects, the online database Gene Expression Profiling Interactive Analysis (GEPIA) was used to analyze the expression of CLCA4 in both colon and rectal cancers using Colon Adenocarcinoma and Rectum Adenocarcinoma datasets of TCGA (21), compared to Match TCGA normal data.

The Human Protein Atlas (THPA) database analysis. THPA, as a database containing images from immunohistochemical (IHC)-based tissue microarrays (TMAs; 46 normal human tissues and 20 types of human cancer) for 11,250 human proteins, was accessed to analyze CLCA4 protein expression in CRC tissues and non-cancerous colorectal tissues, and the images and expression levels of CLCA4 were downloaded from the website (22). Furthermore, Kaplan-Meier survival analysis was performed using log-rank tests via THPA website to analyze the association between CLCA4 expression and the overall survival rate of tumor patients originally sourced from The Cancer Genome Atlas (TCGA) database (cancer. gov/tcga). The auto 'Best expression cut off' was set as cut off for high and low expression of CLCA4 (CRC: High >5.31, low $\leq 5.31$; breast cancer: High $>0.05$, low $\leq 0.05$; head and neck cancer: High $>0.81$, low $\leq 0.81$; stomach cancer: High $>0.02$, low $\leq 0.02$ ).

IHC-based TMA analysis. To assess the protein expression levels of CLCA4 in CRC samples, a TMA (cat. no. HLinAde075Met01) containing colon cancer $(n=8)$ and rectal cancer $(n=6)$ tissues, and their paired non-cancerous colon and rectal tissues was used. To further explore the role of CLCA4 expression during the development of CRC, a TMA (cat. no. HColAde080CD01) with colorectal normal $(n=4)$, adenoma $(n=11)$ and carcinoma $(n=11)$ tissues was used. The tissue specimens for both TMAs were original collected from Taizhou Hospital of Zhejiang Province (Taizhou, China) and tissue collection was approved by its Ethics Committee in accordance with the principles of the Declaration of Helsinki. Both of the aforementioned TMAs were provided by Shanghai Outdo Biotech. IHC was performed to detect CLCA4 protein expression in TMAs using the standard technique (23). Briefly, the sections $(4 \mu \mathrm{m})$ were incubated with a primary antibody against CLCA4 (dilution, 1:100; cat. no. 35684; Signalway Antibody LLC) overnight at $4^{\circ} \mathrm{C}$ after blocking endogenous peroxidase and proteins, and were subsequently incubated with HRP-labeled anti-rabbit secondary antibody (ready to use) for $1 \mathrm{~h}$ at room temperature using UltraSensitive ${ }^{\mathrm{TM}}$ SP IHC kit (cat. no. KIT-9710; Maxim Biomedical, Inc.). At the end of the experiment, the slides were scanned using a NanoZoomer 2.0 HT slide scanner (Hamamatsu Photonics K.K.). The intensity and percentage of positively stained cells were analyzed by experienced pathologists who were blinded to the clinical and pathological data. CLCA4 expression among tissues was analyzed using IHC scores calculated using the following formula: Final score=intensity score $(0$, no staining; 1 , weak; 2 , moderate; and 3 , strong) x percentage score (1, 1-25\% positive; $2,26-50 \%$ positive; $3,51-75 \%$ positive; and $4,76-100 \%$ positive).

Statistical analysis. All statistical tests were conducted using SPSS version 19.0 (IBM Corp.). For the data from GSE49355 dataset and IHC score analysis, 2 related samples comparison were performed with Wilcoxon signed-rank test, and $\mathrm{k}$ independent samples comparisons were analyzed with Kruskal-Wallis test followed by Mann-Whitney U tests and Bonferroni's was used to correct multiple comparisons. $\mathrm{P}<0.05$ was considered to indicate a statistically significant difference.

\section{Results}

Identification of lower CLCA4 $\mathrm{mRNA}$ expression in CRC and metastatic tissues. The NCBI-GEO is a free database of microarray/gene profiles and next-generation sequencing, from which differentially expressed genes between CRC and normal tissues were identified in the GSE49355 dataset. CLCA4 was identified as the only differentially expressed gene between normal colon tissues and primary tumor tissues, and between primary tumors and liver metastases (Fig. 1A). As presented in Fig. 1B, CLCA4 was highly expressed in normal colon tissues, while its expression was significantly downregulated in primary tumor tissues $(\mathrm{P}<0.05$ versus non-cancerous colorectal tissues). Furthermore, CLCA4 mRNA expression was further downregulated in liver metastatic tissues $(\mathrm{P}<0.05$ versus non-cancerous colorectal tissues and versus primary CRC tissues).

Lower CLCA4 mRNA expression in CRC tissues. The Oncomine platform was used to analyze the transcriptional levels of CLCA4 in CRC and other cancer specimens, and in normal controls. As presented in Fig. 2A, CLCA4 mRNA expression was markedly lower in multiple types of cancer tissues, including CRC, than in normal type-matched tissues. The decreases in CLCA4 expression in CRC tissues from 19 Oncomine datasets were presented in Table I (24-28). Furthermore, analyses of CLCA4 expression from the Colon Adenocarcinoma and Rectum Adenocarcinoma datasets of TCGA through the GEPIA website further confirmed the decrease in CLCA4 expression in both colon and rectal cancer tissues (both $\mathrm{P}<0.05$ versus non-cancerous colorectal tissues; Fig. 2B).

Lower CLCA4 protein expression in CRC tissues. The IHC-based THPA database was used to assess CLCA4 protein expression in CRC tissues. CLCA4 protein expression was 
Table I. Oncomine analysis of chloride channel accessory 4 expression in colorectal cancer.

\begin{tabular}{|c|c|c|c|c|}
\hline Cohort & Data type & Sample (n) & Fold-change & P-value \\
\hline Hong Colorectal (24) & mRNA & Colorectal Carcinoma (70) vs. Normal (12) & -594.53 & $3.06 \times 10^{-37}$ \\
\hline \multirow[t]{5}{*}{ Kaiser Colon (25) } & mRNA & Rectosigmoid Adenocarcinoma (10) vs. Normal (5) & -89.63 & $1.88 \times 10^{-10}$ \\
\hline & mRNA & Rectal Adenocarcinoma (8) vs. Normal (5) & -107.16 & $6.54 \times 10^{-9}$ \\
\hline & mRNA & Colon Mucinous Adenocarcinoma (13) vs. Normal (5) & -94.18 & $3.12 \times 10^{-11}$ \\
\hline & mRNA & Cecum Adenocarcinoma (17) vs. Normal (5) & -86.41 & $1.38 \times 10^{-12}$ \\
\hline & mRNA & Colon Adenocarcinoma (41) vs. Normal (5) & -54.90 & $5.91 \times 10^{-14}$ \\
\hline \multirow[t]{2}{*}{ Skrzypczak Colorectal (26) } & mRNA & Colon Adenocarcinoma (45) vs. Normal (24) & -57.90 & $3.59 \times 10^{-14}$ \\
\hline & mRNA & Colorectal Carcinoma (36) vs. Normal (24) & -29.41 & $8.46 \times 10^{-13}$ \\
\hline \multirow[t]{4}{*}{ Skrzypczak 2 Colorectal (26) } & mRNA & Colorectal Carcinoma (5) vs. Normal (10) & -214.32 & $4.80 \times 10^{-9}$ \\
\hline & mRNA & Colon Adenoma (5) vs. Normal (10) & -281.61 & $2.00 \times 10^{-7}$ \\
\hline & mRNA & Colon Adenoma Epithelia (5) vs. Normal (10) & -35.33 & $1.41 \times 10^{-6}$ \\
\hline & mRNA & Colon Carcinoma Epithelia (5) vs. Normal (10) & -36.68 & $1.25 \times 10^{-6}$ \\
\hline \multirow[t]{2}{*}{ Sabates-Bellver Colon (27) } & mRNA & Rectal Adenoma (7) vs. Normal (32) & -51.08 & $6.65 \times 10^{-5}$ \\
\hline & mRNA & Colon Adenoma (25) vs. Normal (32) & -28.15 & $3.99 \times 10^{-9}$ \\
\hline \multirow[t]{4}{*}{ TCGA Colorectal } & mRNA & Cecum Adenocarcinoma (22) vs. Normal (22) & -31.51 & $1.08 \times 10^{-12}$ \\
\hline & mRNA & Rectal Adenocarcinoma (60) vs. Normal (22) & -20.29 & $9.89 \times 10^{-18}$ \\
\hline & mRNA & Colon Adenocarcinoma (101) vs. Normal (22) & -10.47 & $4.55 \times 10^{-14}$ \\
\hline & mRNA & Colon Mucinous Adenocarcinoma (22) vs. Normal (22) & -14.69 & $1.93 \times 10^{-8}$ \\
\hline Gaedcke Colorectal (28) & mRNA & Rectal Adenocarcinoma (65) vs. Normal (65) & -13.91 & $1.03 \times 10^{-20}$ \\
\hline
\end{tabular}

TCGA, The Cancer Genome Atlas.
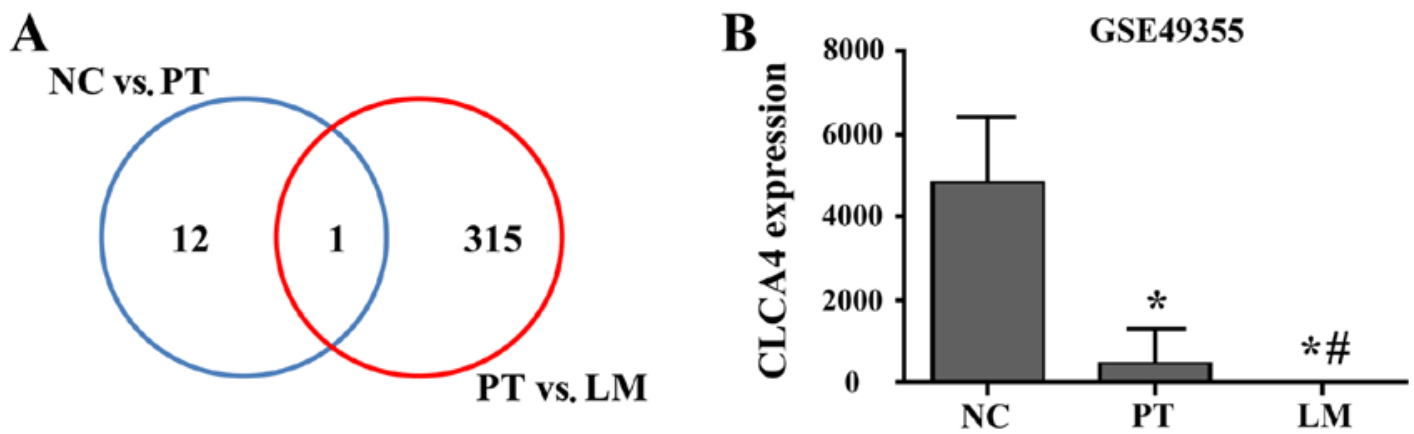

Figure 1. Identification of CLCA4 from microarray data. (A) Differential expression of genes between NC and PT, and between PT and LM in the GSE49355 dataset. (B) CLCA4 expression among NC, PT and LM in the GSE49355 dataset. " $\mathrm{P}<0.05$ vs. NC; ${ }^{\text {P }}<0.05$ vs. PT. NC, normal colon; PT, primary tumor; LM, liver metastases; CLCA4, chloride channel accessory 4.

moderate in three non-cancerous colorectal tissues and was weak $(n=4)$ or not detectable $(n=8)$ in 12 CRC tissues; representative images are presented in Fig. 3. The determination of CLCA4 protein expression from the IHC-based TMAs revealed that CLCA4 protein expression was downregulated in both colon and rectal cancer tissues $(\mathrm{P}<0.05$ versus paired non-cancerous colon or rectal tissues; Fig. 4). The clinical characteristics of the patients with colon and rectal cancer are presented in Table II. The aforementioned experiments revealed that CLCA4 protein expression was downregulated in CRC tissues.

Gradual decrease in CLCA4 expression during the development of $C R C$. To further evaluate CLCA4 expression during the development of CRC, an IHC-based TMA was used to analyze CLCA4 expression in colorectal normal, adenoma and carcinoma tissues. The results revealed that CLCA4 protein expression was downregulated in colorectal adenoma tissues (IHC score: 6.91) compared with normal tissues (IHC score: 11.00), but there was no statistical significance ( $P>0.05$; Fig. 5). Furthermore, CLCA4 protein expression was significantly downregulated in colorectal carcinoma tissues (IHC score: 1.18) compared with colorectal normal tissues and colorecta adenoma tissues, respectively $(\mathrm{P}<0.05$; Fig. 5). The clinical characteristics of the patients were presented in Table III. The present results suggest that a decrease in CLCA4 expression may serve a significant role in the development of CRC.

Low CLCA4 mRNA expression is associated with low overall survival of tumor patients. The association between the 
A

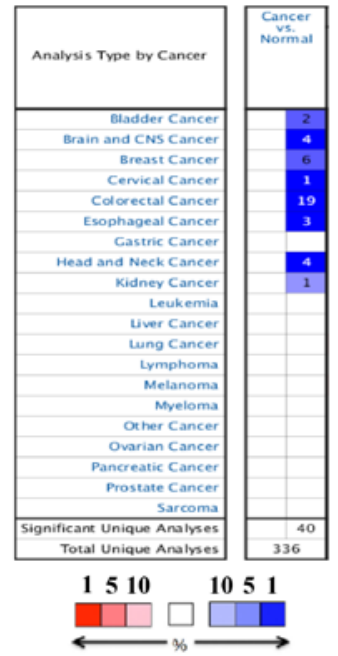

Gene rank percentile
B

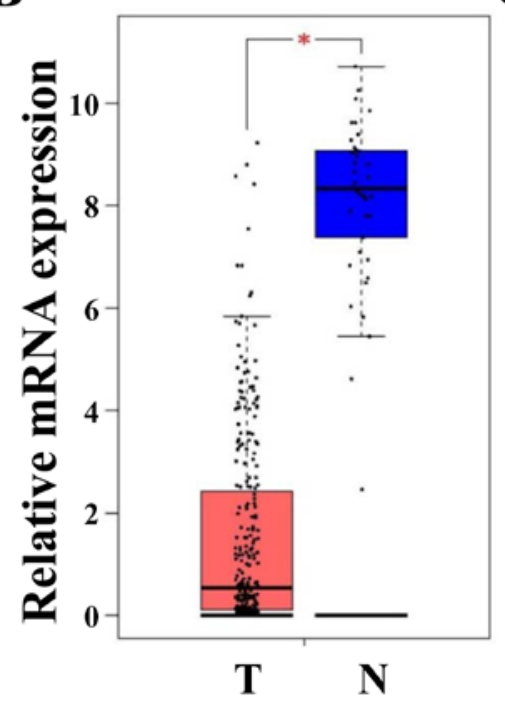

C

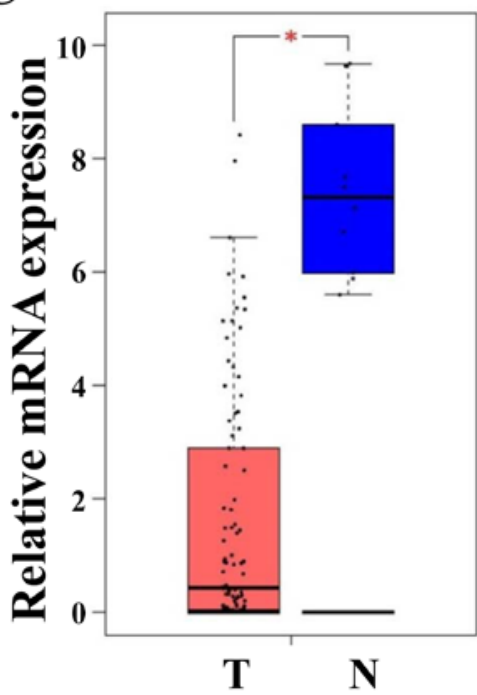

Figure 2. Analyses of CLCA4 mRNA expression through the Oncomine and GEPIA databases. (A) CLCA4 mRNA expression in 20 different types of cancer. Red indicates gene upregulation, and blue indicates gene downregulation. Color transparency indicates a slightly shifted top $1 \%$ to the top $10 \%$ in gene upregulation and downregulation. CLCA4 mRNA expression in (B) colon and (C) rectal cancer tissues was determined by TCGA database analysis through the GEPIA website. ${ }^{*} \mathrm{P}<0.05$. N, normal; T, tumor; CLCA4, chloride channel accessory 4.

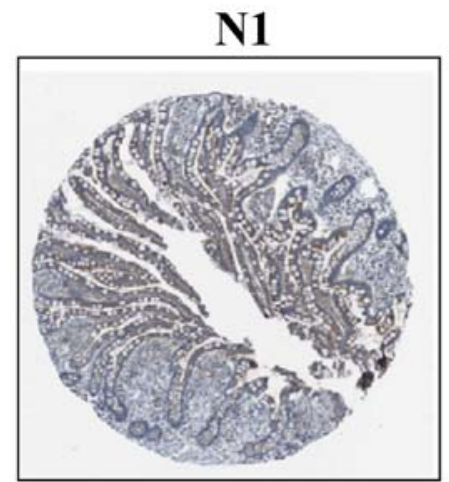

T1

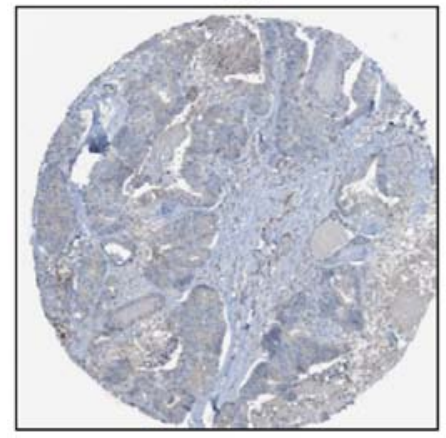

N2

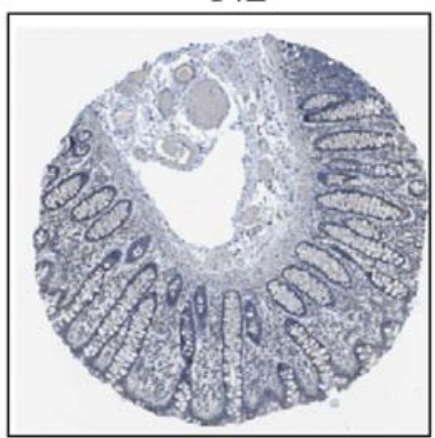

T2

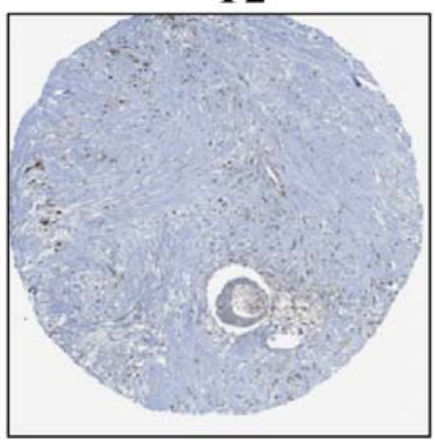

N3

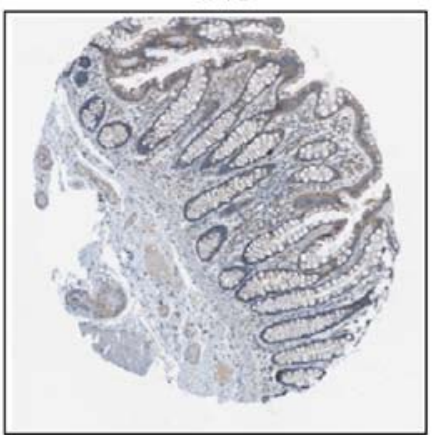

T3

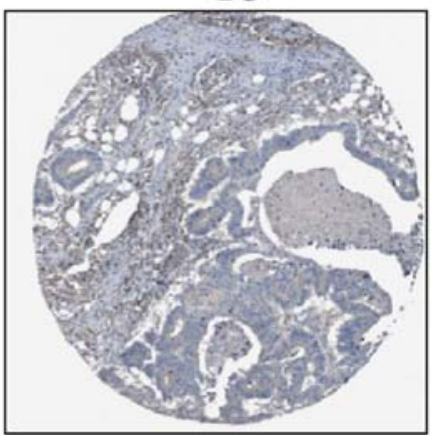

Figure 3. THPA website analysis of CLCA4 protein in CRC. CLCA4 protein expression in CRC specimens and non-cancerous colorectal tissues via THPA website analysis. Three representative images of CRC (https://www.proteinatlas.org/ENSG00000016602-CLCA4/pathology/colorectal+cancer\#img) and non-cancerous colorectal tissues are presented (https:/www.proteinatlas.org/ENSG00000016602-CLCA4/tissue/colon\#img). N, normal; T, tumor; CLCA4, chloride channel accessory 4 ; CRC, colorectal cancer.

expression levels of CLCA4 and the overall survival of patients with CRC was analyzed using the online TCGA dataset through THPA database. As presented in Fig. 6A, overall survival rate of CRC patients was significantly higher in the high CLCA4 expression group than that in the low CLCA4 expression group $(\mathrm{P}<0.05$; cut-off: 5.31). Furthermore, low CLCA4 expression was associated with the shorter overall survival rate of patients with breast cancer $(\mathrm{P}<0.05$; cut-off: 0.05; Fig. 6B), head and neck cancer ( $\mathrm{P}<0.05$; cut-off: 0.81; Fig. $6 \mathrm{C})$ or stomach cancer ( $\mathrm{P}<0.05$; cut-off: 0.02; Fig. 6D). The current results suggested that low CLCA4 expression may be an indicator of poor prognosis in patients with different types of cancer, including CRC. 

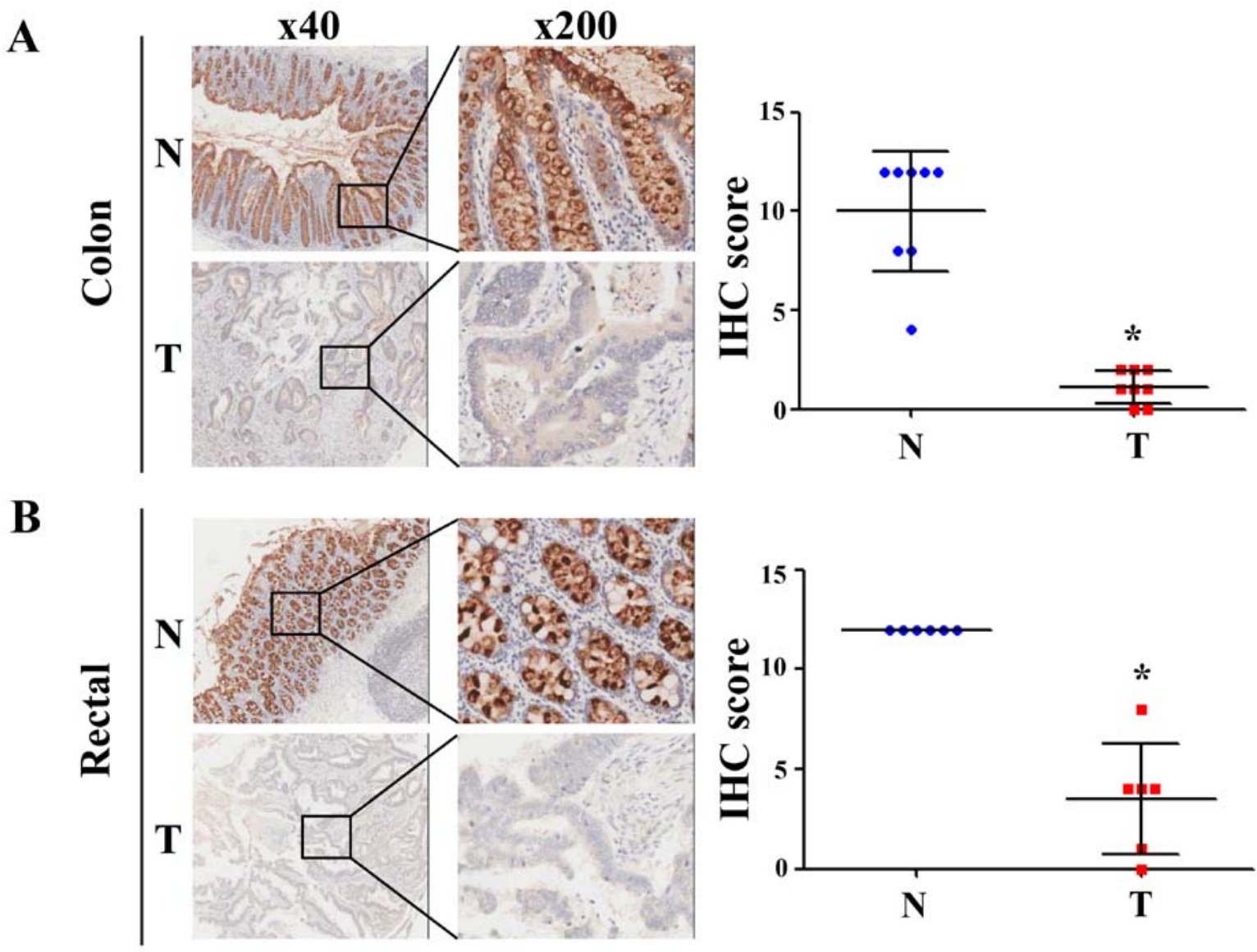

Figure 4. Analysis of CLCA4 protein expression in CRC through IHC-based TMA. IHC-based TMA analysis was conducted to determine the CLCA4 protein expression in (A) colon and (B) rectal cancer tissues, and in their paired non-cancerous tissues. Representative images (magnification, $\mathrm{x} 40$ and $\mathrm{x} 200$ ) are presented in the left panel, while the analyses of protein expression are presented in the right panel. " $\mathrm{P}<0.05$ versus normal colon or normal rectal tissues. $\mathrm{N}$, normal; T, tumor; CLCA4, chloride channel accessory 4; TMA, tissue microarray; IHC, immunohistochemistry.

\section{Discussion}

CRC is the third most prevalent cancer in humans and poses a significant public health problem worldwide (1-4). CRC treatment, despite significant improvements, remains unsatisfactory and CRC prognosis remains poor. In recent decades, systematic analyses of genomic, transcriptomic and proteomic datasets have become powerful tools in the discovery and validation of tumor markers. Therefore, online databases have been analyzed to identify novel genes involved in the development and progression of CRC. To further explore the molecular mechanism involved in the development of CRC, the present study conducted a database analysis and revealed gradual decreases in CLCA4 expression from non-cancerous colorectal tissues to primary and metastatic CRC tissues, suggesting that CLCA4 may act as a tumor suppressor and serve a significant role in the development and progression of CRC.

CLCA4 was a tumor suppressor that contributes to the progression of several types of cancer. In liver cancer, CLCA4 downregulation promoted hepatocellular carcinoma cell proliferation, migration and invasion (14). CLCA4 was aberrantly expressed in breast cancer, and its abnormal expression inhibits tumor cell growth (15). In addition, knockdown of CLCA4 expression causes downregulation of
E-cadherin expression and upregulation of $\mathrm{N}$-cadherin and vimentin expression in breast cancer cells (15). CLCA4 has been identified as a potential therapeutic target for the treatment of oral cancer using bioinformatics analysis (29). In bladder cancer, low CLCA4 expression has been previously associated with larger tumor size, advanced tumor stage and poor prognosis, and CLCA4 overexpression profoundly attenuates the proliferation, growth, migratory and invasive capabilities of bladder cancer cells (16). The aforementioned studies have demonstrated the essential role of CLCA4 in various types of tumor.

In the current study, using the online Oncomine platform, most of the tumor tissues, including those from CRC and brain, breast and esophageal cancer, had significantly lower CLCA4 transcriptional levels compared with those in associated normal tissues. CRC tissues exhibited low CLCA4 expression compared with non-cancerous colorectal tissues from GEPIA datasets. Furthermore, TCGA database and TMA analyses revealed that the CLCA4 mRNA and protein expression was downregulated in both colon and rectal cancer tissues compared with that in non-cancerous colorectal tissues. Analysis from the Oncomine database indicated that CLCA4 mRNA expression was decreased in various types of tumor tissues. The present results revealed that a decrease in CLCA4 expression may be a common event in cancer development, 
Table II. Clinicopathological features of 14 patients with colorectal cancer.

\begin{tabular}{lc}
\hline Characteristic & $\mathrm{n}(\%)$ \\
\hline Age, years & \\
$<65$ & $11(79)$ \\
$\geq 65$ & $3(21)$ \\
Sex & \\
Female & $3(21)$ \\
Male & $11(79)$ \\
Tumor location & \\
Rectum & $6(43)$ \\
Colon & $8(57)$ \\
Clinical stage & \\
I & $0(0)$ \\
II & $12(86)$ \\
III & $2(14)$ \\
IV & $0(0)$ \\
T stage & \\
T1 & \\
T2 & $5(36)$ \\
T3 & $0(0)$ \\
T4 & $8(57)$ \\
N stage & $1(7)$ \\
N0 & \\
N1 & $7(50)$ \\
N2 & $5(36)$ \\
M stage & $2(14)$ \\
M0 & \\
M1 & $12(86)$ \\
Lymph node metastasis & $2(14)$ \\
Yes & \\
No & $6(43)$ \\
\hline & $8(57)$ \\
\hline
\end{tabular}

Table III. Clinicopathological features of 22 patients with colorectal cancer.

\begin{tabular}{lr}
\hline Characteristic & $\mathrm{n}(\%)$ \\
\hline Age, years & \\
$<65$ & $19(86)$ \\
$\geq 65$ & $3(14)$ \\
Sex & \\
Female & $9(41)$ \\
Male & $13(59)$ \\
Tumor type & \\
Colorectal adenocarcinoma & $11(50)$ \\
Colorectal carcinoma & $11(50)$ \\
Clinical stage & \\
I & $5(22)$ \\
II & $14(64)$ \\
III & $3(14)$ \\
IV & $0(0)$ \\
\hline
\end{tabular}
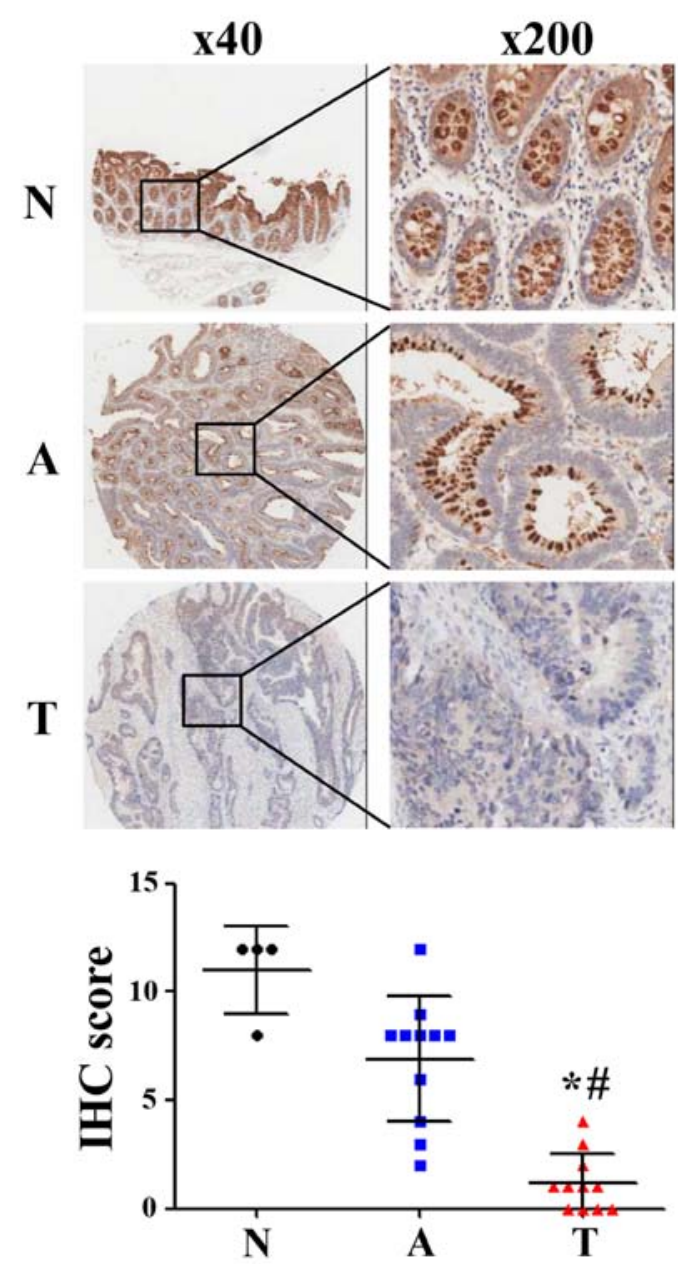

Figure 5. Analysis of CLCA4 protein expression in colorectal adenoma and carcinoma tissues through IHC-based TMA. IHC-based TMA was conducted to determine the CLCA4 protein expression in colorectal normal, colorectal adenoma and colorectal carcinoma tissues. Representative images (magnification, $\mathrm{x} 40$ and $\mathrm{x} 200$ ) are presented in the top panels, while the analysis of protein expression is presented in the bottom panel. ${ }^{*} \mathrm{P}<0.05$ vs. $\mathrm{N}$ tissues; ${ }^{\text {"P}} \mathrm{P}<0.05$ vs. colorectal adenoma tissues. $\mathrm{N}$, colorectal normal; A, colorectal adenoma; T, colorectal carcinoma; CLCA4, chloride channel accessory 4; TMA, tissue microarray; IHC, immunohistochemistry.

including CRC. However, the decrease in CLCA4 expression in other types of tumor tissues should be further evaluated by examining both mRNA and protein levels, and the number of CRC samples should be increased.

To assess CLCA4 expression during the development of CRC, an IHC-based TMA analysis was conducted in the present study. The results demonstrated that CLCA4 protein expression was downregulated in colorectal adenoma tissues compared with normal tissues, but this was not statistical significant, which may have been due to the limited number of samples. Notably, CLCA4 protein expression was significantly downregulated in colorectal carcinoma tissues compared with colorectal normal tissues and colorectal adenoma tissues, respectively. In general, CLCA4 protein expression revealed a gradual decrease in CLCA4 expression among colorectal normal, adenoma and carcinoma tissues, indicating that a decrease in CLCA4 expression may serve a significant role in the development of CRC. Therefore, the 
A

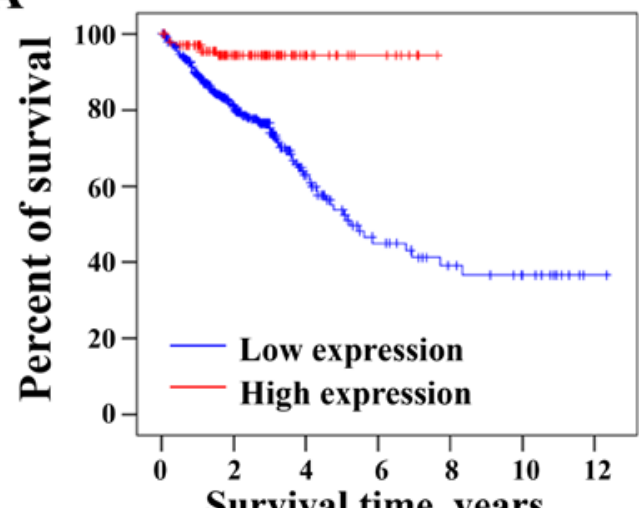

C

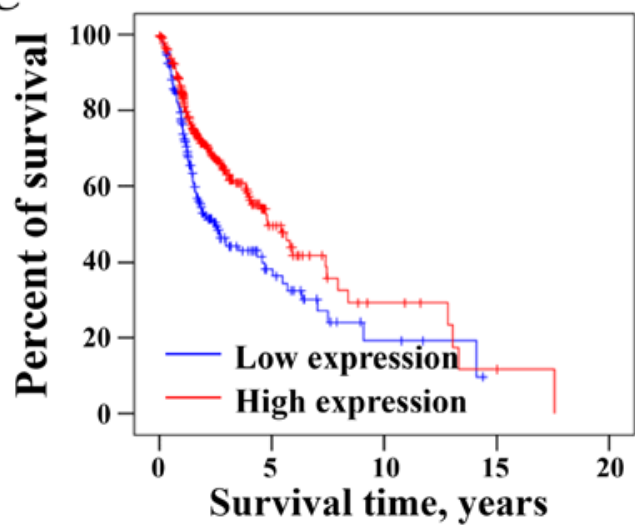

B

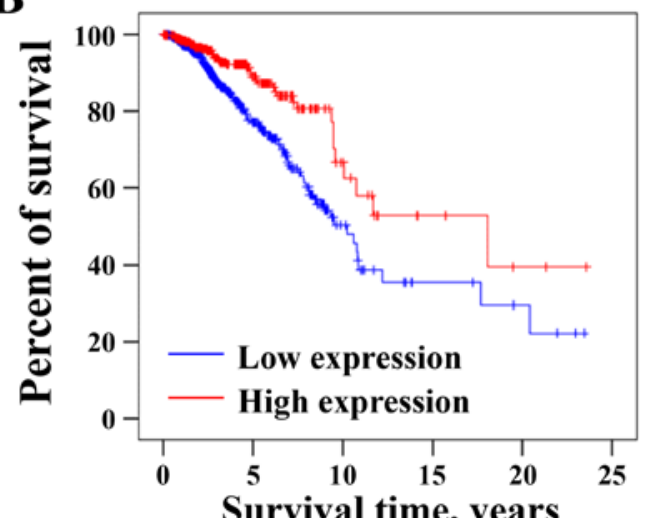

D

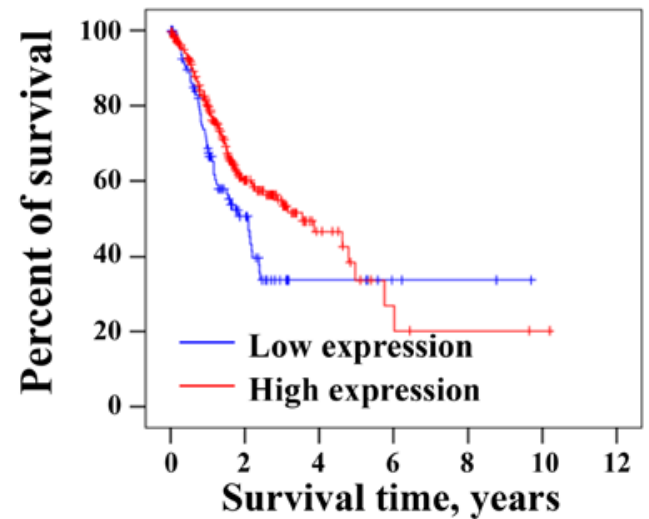

Figure 6. Association between CLCA4 expression and overall survival of patients in different types of tumor. Association between CLCA4 mRNA expression (high or low expression) and overall survival rate of patients with different types of tumor, including (A) colorectal cancer, (B) breast cancer, (C) head and neck cancer and (D) stomach cancer. CLCA4, chloride channel accessory 4.

biological function of CLCA4 during the development of CRC should be further investigated using CLCA4-knockout or -knock-in mice in future studies. In addition, THPA website TCGA survival analysis indicated that low CLCA4 expression was significantly associated with the overall survival of patients with different types of tumor, including CRC, breast cancer, head and neck cancer and stomach cancer, indicating that a decrease in CLCA4 mRNA expression may serve as a prognostic indicator in patients with tumors, including CRC. Furthermore, by conducting an IHC-based TMA analysis, CLCA4 protein expression between primary and metastatic CRC tissues was assessed, but no significant difference was observed (data not shown), which may have been due to the limited number of samples or the difficulty in distinguishing between primary and metastatic CRC tissues. Therefore, the association between CLCA4 expression and CRC metastasis requires a more rational design of high-quality studies to verify the conclusions from the present study. In addition, since the main structure of CLCA4 is similar with CLCA1 and CLCA2 (17), CLCA4 may have the same biological function in regulating cell proliferation and metastasis in $\mathrm{CRC}$, and may regulate these processes by suppressing the $\mathrm{PI} 3 \mathrm{~K} / \mathrm{AKT}$ pathway. Therefore, the concrete mechanism is still remaining to be further elucidated.

To summarize, the present study demonstrated that CLCA4 expression, both in the mRNA and protein levels, was significantly downregulated in CRC tissues compared with that in non-cancerous colorectal tissues. Furthermore, CLCA4 expression was gradually decreased among colorectal normal, adenoma and carcinoma tissues. Additionally, a decrease in CLCA4 expression was significantly associated with the overall survival of patients with different types of tumor, including CRC. Therefore, CLCA4 may serve as a prognostic marker and a potential therapeutic target for CRC. However, CLCA4 expression in different CRC cell lines and its biological functions in cell proliferation and metastasis should be further explored.

\section{Acknowledgements}

Not applicable.

\section{Funding}

The present study was sponsored by the National Natural Science Foundations of China (grant nos. 81774121 and 81703913) and the Natural Science Foundation of Fujian Province, China (grant no. 2017J01846).

\section{Availability of data and materials}

All data generated or analyzed during this study are included in this published article. Microarray dataset of GSE49355 was downloaded from the NCBI-GEO database (https://www. 
ncbi.nlm.nih.gov/geo/query/acc.cgi?acc=GSE49355). The data for difference expression analysis of CLCA4 were obtained from Colon Adenocarcinoma and Rectum Adenocarcinoma datasets of The Cancer Genome Atlas (TCGA) through Gene Expression Profiling Interactive Analysis website (http://gepia.cancer-pku.cn/detail. php?gene=CLCA4). The data for survival analysis were downloaded from TCGA database (https://www.cancer. gov/about-nci/organization/ccg/research/structural-genomics/tcga).

\section{Authors' contributions}

LW, WC and JL conceived and designed the study. LW, WC, JZ and YF performed the data analysis. LW wrote the paper, and JL reviewed and edited the manuscript. All authors read and approved the final manuscript.

\section{Ethics approval and consent to participate}

Tissue collection was approved by the Ethics Committee of Taizhou Hospital of Zhejiang Province (Taizhou, China) in accordance with the principles of the Declaration of Helsinki.

\section{Patient consent for publication}

Not applicable.

\section{Competing interests}

The authors declare that they have no competing interests.

\section{References}

1. Favoriti P, Carbone G, Greco M, Pirozzi F, Pirozzi RE and Corcione F: Worldwide burden of colorectal cancer: A review. Updates Surg 68: 7-11, 2016.

2. Miller KD, Nogueira L, Mariotto AB, Rowland JH, Yabroff KR, Alfano CM, Jemal A, Kramer JL and Siegel RL: Cancer treatment and survivorship statistics, 2019. CA Cancer J Clin 69: 363-385, 2019

3. Dekker E, Tanis PJ, Vleugels JLA, Kasi PM and Wallace MB: Colorectal cancer. Lancet 394: 1467-1480, 2019.

4. Siegel RL, Miller KD and Jemal A: Cancer statistics, 2019. CA Cancer J Clin 69: 7-34, 2019.

5. Brudvik KW, Kopetz SE, Li L, Conrad C, Aloia TA and Vauthey JN: Meta-analysis of KRAS mutations and survival after resection of colorectal liver metastases. Br J Surg 102: 1175-1183, 2015.

6. Zampino MG, Magni E, Ravenda PS, Cella CA, Bonomo G, Della Vigna P, Galdy S, Spada F, Varano GM, Mauri G, et al: Treatments for colorectal liver metastases: A new focus on a familiar concept. Crit Rev Oncol Hematol 108: 154-163, 2016.

7. Elble RC and Pauli BU: Tumor suppression by a proapoptotic calcium-activated chloride channel in mammaryepithelium. J Biol Chem 276: 40510-40517, 2001.

8. Patel AC, Brett TJ and Holtzman MJ: The role of CLCA proteins in inflammatory airway disease. Annu Rev Physiol 71: 425-449, 2009.

9. Piirsoo M, Meijer D and Timmusk T: Expression analysis of the CLCA gene family in mouse and human with emphasis on the nervous system. BMC Dev Biol 9: 10, 2009.

10. Yang B, Cao L, Liu B, McCaig CD and Pu J: The transition from proliferation to differentiation in colorectal cancer is regulated by the calcium activated chloride channel A1. PLoS One 8: e60861, 2013.

11. Sasaki Y, Koyama R, Maruyama R, Hirano T, Tamura M, Sugisaka J, Suzuki H, Idogawa M, Shinomura $Y$ and Tokino T: CLCA2, a target of the p53 family, negatively regulates cancer cell migration and invasion. Cancer Biol Ther 13: 1512-1521, 2012.
12. Qiang YY, Li CZ, Sun R, Zheng LS, Peng LX, Yang JP, Meng DF, Lang YH, Mei Y, Xie P, et al: Along with its favorable prognostic role, CLCA2 inhibits growth and metastasis of nasopharyngeal carcinoma cells via inhibition of FAK/ERK signaling. J Exp Clin Cancer Res 37: 34, 2018.

13. Walia V, Yu Y, Cao D, Sun M, McLean JR, Hollier BG, Cheng J, Mani SA, Rao K, Premkumar L and Elble RC: Loss of breast epithelial marker hCLCA2 promotes epithelial-to-mesenchymal transition and indicates higher risk of metastasis. Oncogene 31: 2237-2246, 2012.

14. Liu Z, Chen M, Xie LK, Liu T, Zou ZW, Li Y, Chen P, Peng X, Ma C, Zhang WJ and Li PD: CLCA4 inhibits cell proliferation and invasion of hepatocellular carcinoma by suppressing epithelial-mesenchymal transition via PI3K/AKT signaling. Aging (Albany NY) 10: 2570-2584, 2018.

15. Yu Y, Walia V and Elble RC: Loss of CLCA4 promotes epithelial-to-mesenchymal transition in breast cancer cells. PLoS One 8: e83943, 2013.

16. Hou T, Zhou L, Wang L, Kazobinka G, Zhang X and Chen Z: CLCA4 inhibits bladder cancer cell proliferation, migration, and invasion by suppressing the PI3K/AKT pathway. Oncotarget 8: 93001-93013, 2017.

17. Loewen ME and Forsyth GW: Structure and function of CLCA proteins. Physiol Rev 85: 1061-1092, 2005.

18. Vogelstein B, Papadopoulos N, Velculescu VE, Zhou S, Diaz LA Jr and Kinzler KW: Cancer genome landscapes. Science 339: 1546-1558, 2013.

19. Del Rio M, Molina F, Bascoul-Mollevi C, Copois V, Bibeau F, Chalbos P, Bareil C, Kramar A, Salvetat N, Fraslon C, et al: Gene expression signature in advanced colorectal cancer patients select drugs and response for the use of leucovorin, fluorouracil, and irinotecan. J Clin Oncol 25: 773-780, 2007.

20. Rhodes DR, Yu J, Shanker K, Deshpande N, Varambally R, Ghosh D, Barrette T, Pandey A and Chinnaiyan AM: ONCOMINE: A cancer microarray database and integrated data-mining platform. Neoplasia 6: 1-6, 2004.

21. Cancer Genome Atlas Network: Comprehensive molecular characterization of human colon and rectal cancer. Nature 487: 330-337, 2012.

22. Uhlen M, Oksvold P, Fagerberg L, Lundberg E, Jonasson K, Forsberg M, Zwahlen M, Kampf C, Wester K, Hober S, et al: Towards a knowledge-based human protein atlas. Nat Biotechnol 28: 1248-1250, 2010

23. Kato T, Hayama S, Yamabuki T, Ishikawa N, Miyamoto M, Ito T, Tsuchiya E, Kondo S, Nakamura Y and Daigo Y: Increased expression of insulin-like growth factor-II messenger RNA binding protein 1 is associated with tumor progression in patients with lung cancer. Clin Cancer Res 13: 434-442, 2007.

24. Hong Y, Downey T, Eu KW, Koh PK and Cheah PY: A 'metastasis-prone' signature for early-stage mismatch-repair proficient sporadic colorectal cancer patients and its implications for possible therapeutics. Clin Exp Metastasis 27: 83-90, 2010.

25. Kaiser S, Park YK, Franklin JL, Halberg RB, Yu M, Jessen WJ, Freudenberg J, Chen X, Haigis K, Jegga AG, et al: Transcriptional recapitulation and subversion of embryonic colon development by mouse colon tumor models and human colon cancer. Genome Biol 8: R131, 2007.

26. Skrzypczak M, Goryca K, Rubel T, Paziewska A, Mikula M, Jarosz D, Pachlewski J, Oledzki J and Ostrowsk J: Modeling oncogenic signaling in colon tumors by multidirectional analyses of microarray data directed for maximization of analytical reliability. PLoS One 5, e13091, 2010.

27. Sabates-Bellver J, Van der Flier LG, de Palo M, Cattaneo E, Maake C, Rehrauer H, Laczko E, Kurowski MA, Bujnicki JM, Menigatti M, et al: Transcriptome profile of human colorectal adenomas. Mol Cancer Res 5: 1263-1275, 2007.

28. Gaedcke J, Grade M, Jung K, Camps J, Jo P, Emons G, Gehoff A, Sax U, Schirmer M, Becker H, et al: Mutated KRAS results in overexpression of DUSP4, a MAP-kinase phosphatase, and SMYD3, a histone methyltransferase, in rectal carcinomas. Genes Chromosomes Cancer 49: 1024-1034, 2010.

29. Bundela S, Sharma A and Bisen PS: Potential therapeutic targets for oral cancer: ADM, TP53, EGFR, LYN, CTLA4, SKIL, CTGF, CD70. PLoS One 9: e102610, 2014.

This work is licensed under a Creative Commons Attribution-NonCommercial-NoDerivatives 4.0 International (CC BY-NC-ND 4.0) License. 